\title{
Metallic Nanostructures Characterized by Three-Dimensional Atom Probe
}

\author{
K. Hono
}

National Institute for Materials Science (NIMS), 1-2-1 Sengen, Tsukuba 305-0047, Japan

The atom probe was originally developed as a tool for surface science; however, from the emerging stage of the technique, metallurgists realized that it could be used to solve many critical problems on the microstructures of metallic materials. By the 1980's, several groups started employing the atom probe technique for microstructural characterizations of metallic materials. In addition to metals and alloys, it has also been applied to a wide variety of materials such as semiconductors and oxide superconductors. However, due to the poor electrical conductivity, the difficulty of specimen preparation, and the uniform microstructural features of these materials, the atom probe technique has not succeed in producing high impact results for non-metallic materials. On the other hand, the atom probe technique has received wide recognition in the metallurgical community, because it was demonstrated that many critical physical metallurgy problems can be solved by the application of the technique. The development of the 3-D atom probe [1] increased the recognition of the atom probe technique even more in the metallurgical and materials science communities, because the direct mapping of atoms in the 3-D real space strongly appealed to the scientists in this area. Now the 3DAP technique has become widespread in more than 20 labs throughout the world, and its number is increasing. There have been several important technical breakthroughs in this community in addition to the development of new detection systems. One important contribution that we cannot overlook is the development of a method for preparing atom probe specimen using the focused ion beam (FIB) technique [2]. This widened the application area of the atom probe technique from bulky metallurgical samples to a wider variety of materials such as thin films, multi-layer thin films, and powders. Another contribution is the recent attempt to operate 3DAP using a femtosecond laser pulse [3], which may lead to an easier operation of the atom probe without specimen rupture and may open a new application area of atom probe in semiconducting and non-conductive materials.

In this talk, I will give an overview on the metallurgical applications of 3DAP by emphasizing the characterizations of metallic nanostructures that are conducted in the author's group. A recent research trend in the metallurgical community is to refine the microstructural scale from the conventional micron order to the nanoscale dimension. When crystal grain size becomes smaller than a few nanometers, where the dislocation activity is lost and the ferromagnetic exchange coupling becomes significant, various unique mechanical and magnetic properties that are not observed in conventional metallic materials appear, e.g. nanocrystalline materials. The characterization of the nanocrystalline materials that are produced by various processing routes can be performed most powerfully by the atom probe technique, because it can reveal atom distributions in three dimensional space. The two dimensional projected information with transmission electron microscopy (TEM) is not sufficient for studying the really important features of nanocrystalline materials. In addition, the capability of light element analysis is important in most nanocrystalline metallic materials, which cannot be performed with the TEM technique.

One widely used method for preparing nanocrystalline materials is the devitrification of amorphous alloys. The Fe-Si-B based nanocrystalline soft magnetic materials that have been successfully adopted in industrial applications are processed by this route. Many investigations have been carried 
out to understand the nanocrystallization mechanism of this type of alloy, and the atom probe technique played a crucial role towards understanding the nanostructure evolution mechanism [4]. The same route is used for processing the nanocomposite hard magnetic materials that are comprised of soft magnetic phases such as $\mathrm{Fe}, \mathrm{Fe}_{3} \mathrm{~B}$ and hard magnetic phases such as $\mathrm{Nd}_{2} \mathrm{Fe}_{14} \mathrm{~B}$ or $\operatorname{Pr}_{2} \mathrm{Fe}_{14} \mathrm{~B}$. Various additional elements are microalloyed in these nanocrystalline materials to optimize the structure and magnetic properties, and the application of 3DAP made substantial contributions towards understanding the roles of these additives [4]. By using the knowledge on the nanostructural evolution mechanism obtained by the 3DAP technique, new alloy compositions were sought and some successful examples of new alloy developments will be presented [5].

3DAP also made substantial contributions in understanding the roles of microalloyed elements in light alloys, in particular aluminum alloys. In the early days of the technique, the atom probe analysis of aluminum alloys were not taken for granted due to its low evaporation field. Therefore, the atom probe characterization of aluminum alloys started much later than the active researches of steels. In aluminum alloys, microalloying is important because it often changes precipitation kinetics, precipitation products and its sequence. A good example of such an effect can be seen in a classical $\mathrm{Al}-\mathrm{Cu}$ alloy with a trace addition of $\mathrm{Ag}$ and $\mathrm{Mg}$ atoms. The precipitation sequence changes by trace additions of $\mathrm{Ag}$ and $\mathrm{Mg}$. The microstructural evolution of this alloy system was followed by 3DAP and a continuous microstructural evolution from solute clusters to plate-like precipitates was successfully monitored using 3DAP [6]. Initially, co-clusters of $\mathrm{Mg}$ and $\mathrm{Ag}$ atoms are formed in the supersaturated solid solution. Then $\mathrm{Cu}$ atoms are incorporated into these clusters forming a plate-like precipitate on the $\{111\}$ planes. The migration of $\mathrm{Ag}$ and $\mathrm{Mg}$ atoms from the interior of the precipitate to the precipitate/matrix interface was observed in the late stage of the precipitate growth with an atomic resolution. To understand the early co-clustering of $\mathrm{Ag}$ and $\mathrm{Mg}$ atoms in view of solute-cluster interactions, coincidence Doppler broadening (CDB) of positron annihilation radiation was also employed along with 3DAP to obtain the information on vacancies with the solute clustering behavior [7]. Due to its industrial importance, major aluminum alloys have now been explored by the 3DAP technique. Its unique power of presenting pre-precipitates clusters has attracted researchers in this area, and now the 3DAP is regarded as the standard analytical technique in the light alloy community. Until recently, there have not been any successful reports on the atom probe analysis of magnesium alloys. Recent studies have demonstrated that magnesium alloys can be characterized with 3DAP with atomic layer resolution [8], and due to the increasing interest on magnesium alloys as light structural materials, more investigations should be published in the coming years.

\section{References}

[1] A. Cerezo, T. J. Godfrey, and G. D. W. Smith, Rev. Sci. Instrum. 59 (1988) 862.

[2] D. J. Larson et al., Ultramicroscopy. 75 (1998) 147.

[3] B. Deconihout et al., Surf. Interf. Analy. (2005) in press.

[4] K. Hono, Prog. Mater. Sci. 47 (2002), 621.

[5] M. Ohnuma et al. J. Appl. Phys. 93 (2003), 9186.

[6] L. Reich, M. Murayama, and K. Hono, Acta Mater. 46 (1998), 6053.

[7] T. Honma et al., Acta Mater. 52 (2004), 1997.

[8] D. H. Ping et al., Phil. Mag. Lett., 82 (2002), 543.

[9] T. Honma et al., Mat. Sci. Eng. A, (2005) in press. 